\title{
Original
}

\section{Ablación del cáncer renal por radiofrecuencia}

\author{
Javier Estebanez Zarranz, Enrique Artozki Morras, Lore Aguirreazaldegui García, \\ Itziar Crespo Crespo, Francisco Bandres Iruretagoyena, Juan P. Sanz Jaka
}

Servicio de Urologia. Hospital Donostia. San Sebastián. España.

\section{Resumen}

Introducción: $\mathrm{El}$ tratamiento estándar de las masas renales de pequeño tamaño es la nefrectomía parcial, que ha demostrado unos resultados oncológicos semejantes a la nefrectomía radical. Recientemente se han desarrollado técnicas de ablación como la radiofrecuencia y la crioterapia, con el fin de minimizar los efectos secundarios de la resección quirúrgica convencional. En este artículo, revisamos la técnica de ablación mediante radiofrecuencia.

Material y métodos: Para esta revisión se ha utilizado la base de datos Medline introduciendo los términos "renal radiofrequency ablation".

Resultados: Se analizan los diferentes sistemas que existen en el mercado para la aplicación de radiofrecuencia. Se describen las técnicas de aplicación (abierta, laparoscópica y percutánea), observando una tendencia clara a la aplicación de forma percutánea. En los estudios clínicos publicados existen ya series de pacientes con seguimientos a medio plazo (3 años) en los que se demuestra unos resultados oncológicos semejantes a los de las técnicas de resección clásicas y con un índice de complicaciones menor.

Conclusiones: La ablación de tumores renales con radiofrecuencia ha demostrado ser un tratamiento eficaz y con mínimas complicaciones. Sin embargo, hasta que no existan seguimientos a largo plazo, se debe utilizar únicamente en pacientes seleccionados.

Palabras clave: Ablación. Radiofrecuencia. Cáncer Renal.

\section{Radiofrequency ablation of Renal Cell Carcinoma}

\section{Abstract}

Introduction: Standard treatment for small renal masses is partial nephrectomy, which has shown oncological results similar to radical nephrectomy. Ablation procedures such as radiofrequency and cryotherapy have recently been developed in order to minimize the side effects of conventional surgical excision. This article reviews radiofrequency ablation.

Materials and methods: For this review, a search was made in the Medline database using the term "renal radiofrequency ablation".

Results: The different currently marketed systems for delivery of radiofrequency energy are examined. The different delivery approaches (open, laparoscopic, and percutaneous) are described. A trend towards use of the percutaneous approach was seen. Published clinical studies already include patient series with mid-term follow-ups (3 years) showing oncological outcomes similar to conventional resection procedures with fewer complications.

Conclusions: Renal tumor radiofrequency ablation has proved to be an effective treatment with minimal complications. However, it should only be used in selected patients until longer follow-up studies are available.

Keywords: Radiofrequency ablation. Renal cell carcinoma.

$\mathrm{S}^{\mathrm{e}}$ ha estimado la incidencia del carcinoma de células renales (CCR) en 150.000 nuevos casos en el mundo al año, representando el $2 \%$ de los tumores malignos ${ }^{1}$. Debido a la utilización masiva de las modalidades diagnósticas por imagen, sobre todo de la ecografía, la mayoría de los CCR actualmente se diagnostican de forma incidental. Independientemente se ha observado un incremento de su incidencia en los países industrializados ${ }^{2}$.
Numerosos estudios han mostrado que los tumores descubiertos incidentalmente tienen un menor grado de diferenciación, estadío y porcentaje de metástasis que los tumores detectados en pacientes sintomáticos ${ }^{3}$.

El tratamiento estándar de estas masas es la nefrectomía parcial, que ha demostrado unos resultados oncológicos semejantes a la nefrectomía radical ${ }^{4}$. Es posible realizar la nefrectomía parcial por vía 
laparoscópica siguiendo los principios oncológicos básicos. En centros especializados y realizada por urólogos expertos ha demostrado obtener los mismos resultados oncológicos que la nefrectomía parcial abierta ${ }^{5}$.

Más recientemente se han desarrollado técnicas de ablación como la radiofrecuencia (RF) y la crioterapia, con el fin de minimizar los efectos secundarios de la resección quirúrgica convencional. Los beneficios potenciales de estos procedimientos serían el descenso de la morbilidad, la menor hospitalización, la rápida vuelta a la actividad normal, la preservación de la función renal, el menor coste y la posibilidad de tratar enfermos con un alto riesgo quirúrgico.

Estas técnicas se pueden realizar mediante punción percutánea guiada por sistemas de imagen como la ecografía, la TAC o la RMN, o bajo visión directa en el curso de una cirugía laparoscópica o abierta. Los resultados preliminares parecen ser prometedores pero necesitan una evaluación con seguimientos a largo plazo. Si estos buenos augurios se confirman, las técnicas ablativas serán en el futuro una clara alternativa a los tratamientos convencionales.

En este artículo, revisamos la técnica de ablación mediante radiofrecuencia. Realizamos comentarios sobre los temas mas controvertidos como las indicaciones, las variantes técnicas y tecnológicas, el seguimiento de los pacientes etc.

\section{MATERIAL Y MÉTODOS}

Para esta revisión se ha utilizado la base de datos Medline introduciendo los terminos "renal radiofrequency ablation”. Se han incluido los artículos en inglés, francés y castellano. Para valorar los diferentes sistemas, se ha revisado la información ofrecida por las diferentes empresas en sus páginas Web.

\section{TECNOLOGÍA}

La ablación por radiofrecuencia es una tecnología relativamente nueva que se utiliza en clínica humana para CCR desde 1976. Consiste en la aplicación de una corriente eléctrica en un rango de radiofrecuencia que produzca la muerte celular. La energía se aplica a través de unas agujas-electrodo diseñadas para este propósito. Esto causa una fricción molecular con producción de calor que genera una desnaturalización de las proteínas celulares y la desintegración de la membrana celular. La necrosis celular se rodea de una zona en la que aprecian cambios inflamatorios. A lo largo del tiempo la zona muestra una progresiva fibrosis con variable reabsorción ${ }^{7}$. El daño producido por la radiofrecuencia depende de la temperatura alcanzada en el tejido y la duración del calor. Temperaturas superiores a $55^{\circ} \mathrm{C}$ durante 4-6 minutos producen un daño celular irreversible ${ }^{8}$ El incrementar la temperatura, puede ser contraproducente ya que temperaturas superiores a $100^{\circ} \mathrm{C}$ producen carbonización y vaporización del tejido, incrementando la impedancia y dificultando el flujo de la corriente eléctrica y por lo tanto limitando el radio de la lesión térmica producida $^{9}$.

El volumen del tejido necrosado depende del calibre y la configuración del electrodo, de la intensidad y duración de la corriente así como de las características del tejido. Por todo esto, se han diseñado diferentes sistemas para aplicar la radiofrecuencia, dependiendo del diseño del electrodo o del control de la intensidad del calor.

\section{Diseño del electrodo}

El diámetro máximo de la lesión térmica producido por una aguja del 17 es de $1.6 \mathrm{~cm}$. Para incrementar el tamaño de la zona necrosada se han utilizado diferentes variantes técnicas. Una es el sistema de Radionics que utilizar 3 agujas standards y las agrupa en una disposición triangular. El sistema de Rita Medical Sistems consiste en una aguja capaz de abrirse en múltiples punzones que se introducen en la lesión. El diseño de LeVeen es parecido pero se expande en forma de paraguas, alcanzando un diámetro de $5 \mathrm{~cm}$., permitiendo tratar tumores de $7 \mathrm{~cm}$. Este es el sistema que se utiliza con el generador de Boston Scientific. También se ha desarrollado un electrodo que permite la infusión de agua directamente en el tejido antes y durante la aplicación de la energía, permitiendo una mejor conducción eléctrica y previniendo el aumento de la impedancia en el tejido. En un estudio comparativo ${ }^{10}$ de los cuatro sistemas realizado en hígado de cerdo, se observa que con el electrodo de perfusión se obtienen los mayores volúmenes de coagulación. Las agujas agrupadas y el diseño de LeVeen producen los volúmenes más esféricos. Las agujas agrupadas y el sistema RITA son los que tienen mayor reproductividad. En la Tabla 1 se describen los 3 sistemas disponibles actualmente. 
Tabla 1. Principales sistemas de Radiofrecuencia comercializados actualmente.

\begin{tabular}{|c|c|c|c|c|c|}
\hline & Electrodo & Potencia & Generador & Agujas & Lesión \\
\hline Tyco & $\begin{array}{l}\text { Cool-tip }{ }^{\mathrm{TM}} \text {. Con } \\
\text { circulación interna } \\
\text { de agua }\end{array}$ & $200 \mathrm{~W}$ & $\begin{array}{l}\text { Controla la } \\
\text { impedancia }\end{array}$ & 17 gauge & Ovoidea de $3 \mathrm{~cm}$ \\
\hline $\begin{array}{l}\text { RITA Medical } \\
\text { Systems }\end{array}$ & $\begin{array}{l}\text { StarBurst } ® . \text { Se abre } \\
\text { en varios punzones }\end{array}$ & $250 \mathrm{~W}$ & $\begin{array}{l}\text { Controla la } \\
\text { temperatura }\end{array}$ & $\begin{array}{l}9 \text { agujas de } \\
\text { diferentes tamaños }\end{array}$ & $\begin{array}{l}\text { Esfericas de } 2 \text { a } 7 \mathrm{~cm} \\
\text { Una aguja: ovoideas de } \\
1 \times 2 \mathrm{~cm}\end{array}$ \\
\hline Boston Scientifics & $\begin{array}{l}\text { LeVeen }^{\circledR} \text {. Se abre en } \\
\text { forma de paraguas }\end{array}$ & $200 \mathrm{~W}$ & $\begin{array}{l}\text { Controla } \\
\text { la impedancia }\end{array}$ & 2 agujas diferentes & Esferica. 2 y $5 \mathrm{~cm}$ \\
\hline
\end{tabular}

\section{Sistemas basados en la temperatura vs sistemas basados en la impedancia}

Los sistemas basados en la temperatura monitorizan la temperatura tisular en la punta del electrodo y emiten suficiente energía para mantener la temperatura tisular a una temperatura predeterminada durante un tiempo predeterminado. Sin embargo, la temperatura en la punta del electrodo puede que no refleje correctamente la temperatura del tejido. Los sistemas basados en la impedancia miden la impedancia del tejido que rodea el electrodo durante el tratamiento. La energía de radiofrecuencia se aplica hasta que se obtiene un nivel de impedancia predeterminada. Un inconveniente de de este sistema es que la temperatura en el área tratada puede que no sea suficiente para producir una necrosis. Además la impedancia tisular varía en los diferentes órganos. Gettman ${ }^{11}$ en un estudio comparativo realizado en riñones de un modelo animal, no encuentra diferencias significativas entre los dos sistemas. Sin embargo en otro estudio experimental en riñones de cerdo, Rehman ${ }^{12}$ encuentra una necrosis completa en los sistemas basados en le temperatura, pero necrosis incompletas en los sistemas basados en la impedancia donde existen áreas con células viables.

Häcker ${ }^{13}$, en un trabajo en el que compara 4 dispositivos diferentes para aplicar radiofrecuencia, obtiene resultados diferentes con cada uno, por lo que concluye que es necesario conocer las características técnicas de cada sistema para alcanzar unos buenos resultados clínicos.

\section{DATOS EXPERIMENTALES Y CAMBIOS PATOLÓGICOS EN ESTUDIOS CLÍNICOS}

En primer lugar hay que resaltar que no existe un buen modelo experimental. La mayoría de los estudios se han realizado en riñón porcino normal o en riñones de ratón con implantación de tumor $V X 2^{14}$. Este es un tumor mucho más agresivo que el CCR humano y por lo tanto poco útil en estudios a largo plazo.

Los cambios inmediatos que se producen en el riñón porcino tras la radiofrecuencia incluyen aumento de la eosinifilia plasmática, pérdida de la integridad del borde celular, borrosidad de la cromatina nuclear y hemorragia interticial ${ }^{7}$. Al tercer día se desarrolla una necrosis cuagulativa con lisis nuclear y un infiltrado inflamatorio entre la zona tratada y el parénquima sano. La degeneración nuclear se completa en el día ${ }^{14}$. Hacia el día 30 la distorsión de la arquitectura de la zona es completa y no se reconocen las características del parénquima renal. El foco necrótico se reabsorbe completamente a los 90 días.

Zlotta $^{6}$ en 1997 realizó un estudio patológico de 2 tumores, uno tratado con radiofrecuencia durante la nefrectomía y otro tratado percutáneamente 1 semana antes de la intervención. En el estudio del riñón extraído se encontró edema estromal y picnosis. En este segundo caso no se encontraron células tumorales viables. Walter ${ }^{15}$ encontró resultados similares en un estudio en 11 casos. Sin embargo existen otros trabajos con resultados diferentes. Michaels ${ }^{16}$ trata 17 casos con ablación percutánea previa a la nefrectomía, encontrando necrosis incompleta en todos los casos. Este autor realiza un estudio de la actividad metabólica de las células tumorales con cepas de NADH. Rendon ${ }^{17}$ encuentra células viables en 4 de 5 tumores en los que hizo la nefrectomía inmediatamente a la radiofrecuencia y en 3 de 6 en los que la nefrectomía la demoró 7 días. De 5 tumores estudiados con esta técnica 4 presentaban células viables. Sin embargo estos trabajos han sido fuertemente criticados en la literatura achacando los fallos en la ablación completa a errores en 
la técnica o fallos en la tecnología ${ }^{18,19}$. El trabajo mas concluyente es el de Matlaga ${ }^{20}$. Se estudian 10 casos tratados con radiofrecuencia y posterior nefrectomía parcial o total demostrándose una necrosis total de los tumores estudiados también con cepas de vitalidad $\mathrm{NADH}$.

\section{INDICACIONES}

A pesar de los buenos resultados obtenidos en las series publicadas, los seguimientos y la cantidad de casos son todavía reducidos. Por lo tanto hasta que no se comuniquen series con seguimientos largos, el tratamiento estándar debe ser la nefrectomía, la nefrectomía parcial o la nefrectomía parcial laparoscópica. Los pacientes que se deben seleccionar para la RF deben ser aquellos que presenten comorbilidades y alto riesgo para la cirugía pero con expectativa de vida mayor de 1 año. Otras indicaciones serían: pacientes monorrenos, tumores múltiples, enfermedad de von Hippel Lindau o función renal limitada.

El tamaño del tumor debe ser menor que el diámetro capaz de necrosar el sistema de RF que se posea. Asímismo ha de tenerse en cuenta la geometría del tumor y de que la zona de ablación debe tener un margen de seguridad. Otro factor a tener en cuenta es la localización del tumor. Los tumores exofíticos rodeados de grasa son los más favorables, ya que las propiedades aislantes de la grasa permiten alcanzar y mantener altas temperaturas. Los tumores centrales o cercanos a los grandes vasos tienen el problema del enfriado que produce la perfusión sanguínea. Para evitar este efecto y realizar mejor la RF, se ha utilizado la embolización previa del tumor ${ }^{21}$.

Las contraindicaciones para la RF incluyen: coagulopatías, infarto de miocardio reciente, angina inestable o proceso agudo como una infección activa severa. La presencia de uréter o intestino a menos de $1 \mathrm{~cm}$. de la zona de ablación debe contraindicar la técnica percutánea pero no la laparoscópica. Hay radiólogos que para evitar estos problemas, instilan agua estéril, solución de dextrosa al $5 \%, \mathrm{CO}_{2}$ o aire entre el tumor y los órganos veci$\operatorname{nos}^{22}$. Cuando la aguja deba atravesar órganos como hígado, bazo o pulmón para llegar al tumor debe también considerarse la opción laparoscópica. Cuando el tumor está muy pegado a la pared abdominal o al diafragma pueden existir lesiones musculares y nerviosas que condicionen dolor postoperatorio intenso ${ }^{23}$.

\section{TÉCNICA}

Uno de los desafíos más importantes de la ablación con RF es la elección en la forma de aplicarla y la monitorización de la lesión durante el tratamiento. Se ha aplicado RF en los CCR por vía abierta y vía laparoscópica, pero la evolución de la tecnología nos empujará a aplicarla por la vía menos agresiva, la vía percutánea.

\section{Técnica laparoscópica}

La forma primaria de colocación de los electrodos en el los tumores exofíticos es visual. Se introduce la aguja en el centro del tumor y el cirujano observa su ablación con un margen de por lo menos $5 \mathrm{~mm}$. Los ultrasonidos pueden usarse para confirmar la buena colocación de la aguja en el interior del tumor, pero no son eficaces para valorar la extensión de la ablación. En modelos animales y humanos, el área que se observa lesionada con los ultrasonidos, no corresponde con el tamaño real de la lesión 24,25 . Otros grupos han encontrado dificultades con los ultrasonidos debido a las microburbujas que se crean durante el calentamiento tisular ${ }^{26}$. Utilizando un aparato de ultrasonidos tridimensional realzado con contraste, se mejora la correlación entre el resultado de la ultrasonografía y el tamaño real de la ablación en modelos animales ${ }^{27,28}$. Sin embargo, la mayoría de autores opina que la imagen de la ablación en tiempo real, no es necesaria ya que el diámetro de la ablación viene definido por el sistema de agujas que se utiliza.

\section{Técnica percutánea}

La ablación percutánea por RF se puede realizar bajo sedación intravenosa, aunque algunos radiólogos prefieren realizarla con anestesia general. La cuestión de realizar biopsia previa y cuando realizarla es controvertida. Hay autores que piensan que no hay que hacerla y otros que no hacen una ablación sin un diagnóstico previo y hay autores que hacen la biopsia el mismo día de la ablación.

Para guiar el procedimiento se ha utilizado los ultrasonidos, la TAC o la RMN. Cada uno de ellos tiene sus ventajas y sus desventajas. La RMN es el sistema que mas exactamente monitoriza el efecto de la ablación y nos muestra que ha sido correctamente realizada. Es capaz de mostrar imágenes de tumor viable residual inmediatamente después de la ablación, lo que da al radiólogo la opción de realizar un nuevo tratamiento y por lo tanto incrementando 
los buenos resultados. Lewin ${ }^{29}$ utilizando este sistema trata 10 pacientes sin encontrar recidivas en 25 meses de seguimiento. Sin embargo la habilitación de la RMN para guiar la ablación es limitada, ya que se precisa de un hardware accesorio y un software suplementario así como un equipo especial para monitorizar al paciente. Los ultrasonidos facilitan una perfecta visualización de la aguja dentro del tumor, pero como ya se ha dicho antes no son buenos para valorar la correcta ablación. Además algunos tumores no se aprecian correctamente por ecografia. Al igual que los ultrasonidos la TAC no es capaz de precisar los márgenes de la zona de ablación. Sin embargo proporciona mejores datos a la hora de planificar el tratamiento, basándonos en el tamaño y geometría del tumor. Esto permite elegir la posición del electrodo y el tipo de electrodo, que determina la geometría y el tamaño de la zona de ablación.

\section{CONTROL POR IMAGEN POST-ABLACIÓN}

Debido a la ausencia de estudio patológico de la pieza extirpada, la utilización de técnicas de imagen es fundamental para la valoración de los resultados de la ablación por RF. El dato mas importante es que las áreas de necrosis no muestran realce en la TAC o la RMN y sin embargo las áreas de tumor viable si que lo muestran. Este esquema de interpretación se basa en los estudios de correlación radiológico-patológico realizados por Goldberg ${ }^{30}$ en tumores de hígado.

Existen dos lesiones asociadas que se aprecian frecuentemente en la evolución de los tumores tratados con RF. Se trata de un halo en la grasa peritumoral, cuyo significado parece ser una reacción fibrosa en el borde de la zona de ablación en la grasa perirrenal. También se puede apreciar en la involución del tumor, una infiltración grasa que se interpone entre el tejido tratado y el parénquima sano. Mattsumoto ${ }^{31}$ lo encuentra en el $50 \%$ de los casos.

Los intervalos de los controles postoblación están en discusión. Algunos autores realizan el primer control a la semana. Otros esperan un mes. En este primer control hay que buscar cualquier lesión sospechosa de tumor viable para programar la repetición del procedimiento. Después se suelen realizar controles a los 3, 6 y 12 meses y posteriormente anual o bianual dependiendo de la comorbilidad del paciente $^{32}$. La mayoría de los tumores muestran algo de involución, pero a largo plazo no se observa la completa reabsorción que se ve en los animales de experimentación.

\section{ESTUDIOS CLÍNICOS}

Las principales series se resumen en la Tabla 2. McGovern ${ }^{33}$ publica los primeros casos de utilización de la RF como tratamiento del CCR. En esta primera serie de 9 tumores en 8 pacientes con un seguimiento de 10 meses no se observan recidivas tumorales.

Matsumoto ${ }^{34}$ presenta una serie de $109 \mathrm{CCR}$ en 91 pacientes tratados con $\mathrm{RF}$ mediante punción TAC-guiada $(\mathrm{n}=63)$ y laparoscópica $(\mathrm{n}=46)$. La media del tamaño tumoral era de $2.4 \mathrm{~cm}$ (rango: 0,8-4,7). En 107 tumores se obtuvo una ablación completa en la primera sesión y únicamente dos casos precisaron de una nueva sesión. En el grupo de pacientes con un seguimiento de más de 1 año, sólo se comunica una recidiva local $(1,7 \%)$ sin ningún caso de progresión a distancia. La recurrencia local fue tratada también con ablación.

Zagoria $^{35}$ presenta una serie de 125 CCR en 104 pacientes tratados percutáneamente bajo anestesia local y sedación. El tamaño tumoral medio era de $2,7 \mathrm{~cm}$. (rango: 0,6-8,8). Utiliza un generador de 200W controlado por la impedancia y electrodos con irrigación de agua. El seguimiento medio fue de 13,8 meses (rango: 1-75,8). Todos los tumores menores de $3,7 \mathrm{~cm}$ fueron tratados completamente pero cuando el tumor era mayor se apreciaba un tratamiento incompleto en un 30\% de los casos. Los pacientes fueron dados de alta el mismo día del tratamiento y solo se presentaron complicaciones en un $8 \%$ de los casos (ninguna de ellas significó morbilidad a largo plazo).

Gervais $^{36}$ trata 100 tumores en 85 pacientes durante 6 años. Los tumores medían $3,2 \mathrm{~cm}$ de media (rango: 1,1-8,9). 68 tumores eran exofiticos y fueron correctamente tratados. Sin embargo solo se obtiene buena ablación en 7 de 9 casos (78\%) de tumores centrales y en 11 de 18 (61\%) tumores mixtos (con componente periférico y central). También tiene unos peores resultados en tumores grandes. Con una sesión de ablación obtiene una necrosis total en el $100 \%$ de los tumores menores de $3 \mathrm{~cm}$. Sin embargo esto sólo se obtiene en el 53\% de los tumores entre 3 y $5 \mathrm{~cm}$ y en el $28 \%$ de los tumores de más de $5 \mathrm{~cm}$.

En la literatura española sólo se encuentra una serie publicada por el equipo del Hospital de Navarra ${ }^{37}$. Se presentan 4 tumores en 3 pacientes tratados 1 por laparoscopia y 3 percutáneos bajo control ecográfico y con anestesia general. Se utiliza 
Tabla 2. Principales series publicadas

\begin{tabular}{|c|c|c|c|c|c|c|}
\hline Autor & $\begin{array}{l}\text { Tumores/ } \\
\text { pacientes }\end{array}$ & $\begin{array}{l}\text { Tamaño } \\
(\mathrm{cm})\end{array}$ & Localización & Técnica & Resultados & Complicaciones \\
\hline Farell & $35 / 20$ & $\begin{array}{l}0,9-3,6 ; \\
\text { media: } 1,7\end{array}$ & $\begin{array}{l}22 \text { exofíticos, } \\
13 \text { centrales }\end{array}$ & $\begin{array}{l}\text { Percutánea } \\
\text { (US y TAC) } \\
\text { Abierta }\end{array}$ & $35 / 35(100 \%)$ & 1 Dolor por lesión en plexo lumbar \\
\hline Mayo-Smith & $32 / 32$ & $\begin{array}{l}1-0-5,0 ; \\
\text { media: } 2,6\end{array}$ & $\begin{array}{l}29 \text { exofiticos, } \\
3 \text { mixtos }\end{array}$ & $\begin{array}{l}\text { Percutánea } \\
\text { (US y TAC) }\end{array}$ & $31 / 32(97 \%)$ & 1 Metástasis en piel \\
\hline Ogan & $16 / 15$ & $\begin{array}{l}1,4-3,6 ; \\
\text { media: } 2,4\end{array}$ & $\begin{array}{l}10 \text { exofiticos, } \\
2 \text { centrales, } \\
1 \text { mixto }\end{array}$ & $\begin{array}{l}\text { Percutánea } \\
\text { (TAC) }\end{array}$ & $12 / 13(93 \%)$ & 0 \\
\hline Matsumoto & $109 / 91$ & $\begin{array}{l}0,8-4,7 ; \\
\text { media: } 2,4\end{array}$ & & $\begin{array}{l}\text { Percutánea } \\
\text { (TAC) } \\
\text { Laparoscopia }\end{array}$ & & \\
\hline Lewin & $10 / 10$ & $\begin{array}{l}\text { 1,0-3,6; } \\
\text { media: } 2,3\end{array}$ & 10 exofiticos & $\begin{array}{l}\text { Percutánea } \\
(\mathrm{RMN})\end{array}$ & $10 / 10(100 \%)$ & 0 \\
\hline Varkarakis & $56 / 46$ & $\begin{array}{l}\text { 1,0-4,0; } \\
\text { media: } 2.2\end{array}$ & $\begin{array}{l}39 \text { exofiticos } \\
17 \text { centrales }\end{array}$ & $\begin{array}{l}\text { Percutánea } \\
\text { (TAC) }\end{array}$ & $50 / 56(89 \%)$ & 1 neumonía por aspiración $\rightarrow$ fallecido \\
\hline Gervais & $100 / 85$ & $\begin{array}{l}\text { 1,1-8,9; } \\
\text { media: } 3,2\end{array}$ & $\begin{array}{l}68 \text { exofiticos } \\
18 \text { mixtos } \\
9 \text { centrales } \\
5 \text { parenquima }\end{array}$ & $\begin{array}{l}\text { Percutánea } \\
\text { (TAC) }\end{array}$ & $90 / 100(90 \%)$ & $\begin{array}{l}2 \text { hemorragias mayores, } 1 \text { masa } \\
\text { inflamatoria en el tracto, } 2 \text { dolor } \\
\text { lumbar, } 2 \text { lesiones de uréter, } \\
1 \text { quemadura de piel }\end{array}$ \\
\hline Breen & $105 / 97$ & $\begin{array}{l}1,1-6,8 \\
\text { media: } 3,2\end{array}$ & & $\begin{array}{l}\text { Percutánea } \\
\text { (US y TAC) }\end{array}$ & $(90,5 \%)$ & $\begin{array}{l}1 \text { hematuria, } 1 \text { lesión duodenal, } \\
1 \text { estenosis de uréter, } 1 \text { neumotórax, } \\
1 \text { fístula calicial }\end{array}$ \\
\hline Arzola & $27 / 23$ & media: 2,6 & & $\begin{array}{l}\text { Percutánea } \\
\text { (TAC) }\end{array}$ & $18 / 20(90 \%)$ & 0 \\
\hline Salagierski & $45 / 42$ & $\begin{array}{l}1,8-5,9 \\
\text { media: } 3,7\end{array}$ & & $\begin{array}{l}\text { Percutánea } \\
\text { (US y TAC) }\end{array}$ & $45 / 42(93 \%)$ & 0 \\
\hline
\end{tabular}

un sistema de control por la impedancia y agujas de LeVeen. No hay complicaciones y el seguimiento es de 9 meses.

Hay un trabajo comparativo entre la técnica de ablación percutánea y la ablación laparoscópica 38 . Concluye, como era de esperar, que la técnica percutánea se asocia a un postoperatorio más confortable.

Un estudio del equipo de la Universidad de Texas $^{39}$ compara un grupo de pacientes tratados con nefrectomía parcial (abierta o laparoscópica) y otro grupo tratado con ablación por RF (percutánea o laparoscópica). Con un seguimiento de 3 años, los grupos no muestran diferencias significativas en cuanto a los resultados oncológicos.

En la Cleveland Clinic ${ }^{40}$ han hecho una comparación entre un grupo de ablación por crioterapia por vía laparoscópica y otro tratado con ablación percutánea con RF. En el grupo de RF se observa una mayor persistencia o recurrencia radiológica del tumor, pero la mayoría de ellos fueron fácilmen- te retratados con la misma técnica, lo que ofrece una ventaja sobre la crioablación. El seguimiento de los pacientes es corto, por lo que los resultados oncológicos no son valorables. A destacar que el índice de complicaciones mayores es mas alto con la crioablación laparoscópica.

\section{COMPLICACIONES}

En un estudio multicentrico ${ }^{41}$ se estableció una tasa de complicaciones para la ablación con RF de $8,3 \%$ de las cuales $6 \%$ fueron consideradas menores y un 2,3\% mayores. Se describió una muerte por una neumonía por aspiración. En general la tasa de complicaciones para la RF es favorable si la comparamos con las tasas publicadas para la nefrectomía parcial abierta ${ }^{42}(13,7 \%)$ o la nefrectomía parcial laparoscópica $^{43}$ (33\%). Además hay que tener en cuenta que los pacientes seleccionados para realizar las técnicas ablativas, son habitualmente pacientes de alto riesgo quirúrgico. 
La mayoría de las series comunican hematomas subcapsulares o perinefríticos en sus complicaciones, pero la mayoría son valoradas como complicaciones menores, ya que no requieren ningún tipo de intervención. Otra complicación frecuente es el dolor. La mayoría cede con analgesia habitual y es rara la readmisión hospitalaria por este problema. En un trabajo realizado por Baker ${ }^{44}$ sobre 46 pacientes se observó que la necesidad de analgesia dependía de la proximidad de la masa a la pared muscular.

Más importantes son las complicaciones derivadas de la lesión de órganos contiguos cuando la técnica se realiza percutáneamente. Se han descrito lesiones de uréter que producen fístulas o estenosis, la mayoría de ellas tratadas con la colocación de un catéter ureteral. Más peligrosas son las lesiones intestinales que siempre acarrean problemas importantes, incluso la muerte del paciente. Por eso hay autores ${ }^{45}$ que aconsejan realizar la ablación por vía laparoscópica cuando el tumor esté cerca del intestino. Como describimos en la Tabla 1 hay autores que refieren complicaciones menos frecuentes como neumotorax, metástasis en la zona del trayecto de la punción, etc.

\section{CONCLUSIONES}

La ablación por radiofrecuencia de los CCR ha demostrado ser una técnica eficaz y con mínimas complicaciones. Las series publicadas tienen resultados a medio plazo comparables con las técnicas clásicas de resección de las masas renales. Queda resolver el problema de las diferentes tecnologías, ya que se han observado diferentes resultados. Se necesitan estudios comparativos entre ellas. Asimismo se precisan valorar series de pacientes a largo plazo para poder ser incluido como un tratamiento de primera línea. Hay que tener en cuenta que los buenos resultados oncológicos deben estar mitigados por la probabilidad de que algunas de estas masas pueden ser benignas y las que son malignas suelen ser bastante indolentes Mientras esto no esté resuelto, esta técnica debe aplicarse únicamente en pacientes seleccionados.

\section{REFERENCIAS}

1. Godley P, Kim SW. Renal cell carcinoma. Curr Opin Oncol. 2002; 14(3):280-285.

2. Chow WH, Devesa SS, Warren JL, Fraumeni JF Jr. Rising incidence of renal cell cancer in the United States. JAMA. 1999; 281(17):1628-1631.

3. Congregado B, Medina RA, Sanchez E, Morales A, Pascual JL. Diagnostico incidental del carcinoma renal. ¿Implica un mejor pronóstico? Actas Urol Esp. 2001;25(4):278-282.

4. Novick AC. Nephron-sparing surgery for cell carcinoma. Annu Rev Med. 2002;53:393-407.
5. Moinzadeh A, Gill IS, Finelli A, Kaouk J, Desai M. Laparoscopic partial nephrectomy: 3-year follow-up. J Urol. 2006;175(2): 559462

6. Zlotta AR, Wilschutz T, Raviv G, Peny MO, van Gansbeke D, Noel JC, et al. Radiofrequency interstitial tumor ablation (RITA) is a possible new modality for treatment of renal cancer: ex vivo and in vivo experience. J Endourol. 1997;11(4):251-258.

7. Hsu TH, Fidler ME, Gill IS. Radiofrequency ablation of the kidney: acute and chronic histology in porcine model. Urology. 2000;56(5):872-875.

8. McAchran SE, Lesani OA, Resnick MI. Radiofrequency ablation of renal tumors: past, present, and future. Urology. 2005;66(5): 15-22.

9. Gervais DA, Arellano RS, Mueller PR. Percutaneous radiofrequency ablation of renal cell carcinoma. Eur Radiol. 2005;1(5): 960-967.

10. Pereira PL, Trübenbach J, Schenk M, Subke J, Kroeber S, Schaefer I, et al. Radiofrequency ablation: In vivo comparison of four commercially available devices in pig livers. Radiology. 2004;232(2):482-490.

11. Gettman MT, Lotan Y, Corwin TS, Smith TG, Napper CA, Lindberg G, Cadeddu JA. Radiofrequency coagulation of renal parenchyma: comparison of effects of energy generators on treatment efficacy. J Endourol. 2002;16(2):83-88.

12. Rehman J, Landman J, Lee D, Venkatesh R, Bostwick DG, Sundaran C, Clayman RV. Needle-based ablation of renal parenchyma using microwave, cryoablation, impedance- and temperature-based monopolar and bipolar radiofrequency, and liquid and gel chemoablation: laboratorystudies and review of the literature. J Endourol. 2004;18(1):83-104.

13. Häcker A, Vallo S, Weiss C, Grobholz R, Alken P, Knoll T, et al. Minimally invasive treatment of renal cell carcinoma: comparison of 4 different monopolar radiofrequency devices. Eur Urol. 2005;48(4):584-592.

14. Eichel L, Kim IY, Uribe C, Khonsari S, Basillote J, Steward E, et all. Third Prize: Comparison of radical nephrectomy, laparoscopic microwave thermotherapy, cryotherapy, and radiofrequency ablation for destruction of experimental VX-2 renal tumors in rabbits. J Endourol. 2005;19(9):1082-1087.

15. Walther MM, Shawker TH, Libutti SK, Lubensky I, Choyke PL, Venzon D, et al. A phase 2 study of radiofrequency interstitial tissue ablation of localized renal tumors. J Urol. 2000;163(5): 14241427.

16. Michaels MJ, Harrison KR, Moyrtzinos AP, Summerhayes IC, Silverman ML, Libertino JA. Incomplete renal tumor destruction using radiofrequency interstitial ablation. J Urol. 2002; 168(6):2406-2410.

17. Rendon RA, Kachura JR, Sweet JM, Gertner MR, Sherar MD, Robinette $\mathrm{M}$, et al. The uncertainty of radiofrequency treayment of renal cell carcinoma: findings at immediate and delayed nephrectomy. J Urol. 2002;167(4):1587-1592.

18. Ogan K, Cadeddu JA. Re: the uncertainty of radiofrequency treayment of renal cell carcinoma: findings at immediate and delayed nephrectomy. J Urol. 2002;168(5):2128.

19. Rehman J, Landman J, Sundaran CP. Re: the uncertainty of radiofrequency treayment of renal cell carcinoma: findings at immediate and delayed nephrectomy. J Urol. 2002;168(5): 21282129.

20. Matlaga BR, Zagoria RJ, Woodruff RD, Torti FM, Hall MC. Phase II trial of radio frequency ablation of renal cancer: evaluation of the kill zone. J Urol. 2002;168(6):2401-2405.

21. Arima K, Yamakado K, Kimbara H, Nakatsuka A, Takeda K, Sugimura Y. Percutaneous radiofrequency ablation with transarterial embolization is useful for treatment of stage 1 renal cell carcinoma with surgical risk: results at 2-year mean follow up. Int J Urol. 2007;14(7):585-590. 
22. Gervais DA, Arellano RS, McGovern FJ, McDougal WS, Mueller PR. Radiofrequency ablation of renal cell carcinoma: part 2, lessons learned with ablation of 100 tumors. AJR. 2005; 185(1):72-80.

23. Anderson JK, Shingleton WB, Cadeddu JA. Imagin associated with percutaneous and intraoperative management of renal tumors. Urol Clin North Am. 2006;33(3):339-352.

24. Gill IS, Hsu TH, Fox RL, Matamoros A, Miller CD, Leveen RF, et al. Laparoscopic and percutaneous radiofrequency ablation of the kidney: acute and chronic porcine study. Urology. 2000;56(2): 197-200.

25. Gervais DA, McGovern FJ, Wood BJ, Goldberg SN, McDougal WS, Mueller PR. Radiofrequency ablation of renal cell carcinoma: early clinical experience. Radiology 2000;217(3):665-672.

26. Wagner AA, Solomon SB, Su LM. Tretmant of renal tumors with radiofrequency ablation. J Endourol. 2005;19(6):643-652.

27. Johnson DB, Duchene DA, Taylor GD, Pearle MS, Cadeddu JA. Contrast-enhanced ultrasound evaluation of radiofrequency ablation of the kidney: reliable imaging of the thermolesion. $J$ Endourol. 2005;19(2):248-252.

28. Slabaugh TK, Machaindze Z, Hennigar R, Ogan K. Monitoring radiofrequency renal lesions in real time using contrast-enhanced ultrasonography: a porcine model. J Endourol. 2005;19(5): 579-583.

29. Lewin JS, Nour SG, ConnellCF, Sulman A, Duerk JL, Resnick MI, et al. Phase II clinical trial of interactive MR imaging-guided interstitial radiofrequency thermal ablation of primary kidney tumors : initial experience. Radiology. 2004;232(3):835845.

30. Goldberg SN, Gazelle GS, Compton CC, Mueller PR, Tanabe KK. Treatment of intrahepatic malignancy with radiofrequency ablation: radiologic-pathologic correlation. Cancer. 2000;88 (11):2452-2463.

31. Matsumoto ED, Watumull L, Johnson DB, Ogan K, Taylor GD, Josephs S, et al. The radiographic evolution of radio frequency ablated renal tumors. J Urol. 2004;172(1):45-48.

32. Gervais DA, McGovern FJ, Arellano RS, McDougal WS, Mueller PR. Renal cell carcinoma: clinical experience and technical success with radiofrequency ablation of 42 tumors. Radiology. 2003;226(2):417-424.

33. McGovern FJ, Wood BJ, Goldberg SN, Mueller PR. Radiofrequency ablation of renal cell carcinoma via image guided needle electrodes. J Urol. 1999;161(2):665-672.

34. Matsumoto ED, Johonson DB, Ogan K, Trimmer C, Sagaloowsky A, Margulis V, Cadeddu JA. Short-term efficacy of temperature-based radiofrequency ablation of small renal tumors. Urology. 2005;65(5):877-881.
35. Zagoria RJ, Traver MA, Werler DM, Perini M, Hayasaka S, Clark PE. Oncologic efficacy of CT-guided percutaneous radiofrecquency ablation of renal cell carcinomas. AJR. 2007;189(2): 429-436.

36. Gervais DA, McGovern FJ, Arellano RS, McDougal WS, Mueller PR. Radiofrequency ablation of renal cell carcinoma: part 1, Indications, results, and role in patient management over a 6year period and ablation of 100 tumors. AJR. 2005;185(1):6471.

37. Pascual Piédrola JI, Grasa Lanau V, Aldave Villanueva J, Arrondo Arrondo JL, Cuesta Alcalá JA, Ponz González M, et al. Experiencia inicial en la ablación percutánea conradiofrecuencia de los tumores renales. Arch Esp Urol. 2004;57(10):1113-1119.

38. Bandi G, Hedican S, Moon T, Lee FT, Nakada SY. Comparison of postoperative pain, convalescence, and patient satisfaction after laparoscopic and percutaneous ablation of renal masses. J Endourol. 2008;22(5):963-967.

39. Stern JM, Svatec R, Park S, Hermann M, Lotan Y, Sagalowsky $\mathrm{AI}$, Cadeddu JA. Intermediate comparison of partial nephrectomy and radiofrequency ablation for clinical Tla renal tumours. BJU Int. 2007;100(2):287-290.

40. Hegarty NJ, Gill IS, Desai MM, Remer EM, O`Malley CM, Kaouk JH. Probe-ablative nephron-sparing surgery: cryoablation versus radiofrequency ablation. Urology. 2006;68(1 Suppl):7-13.

41. Johnson DB, Solomon SB, Matsumoto ED, Kavoussi LR, Nakada SY, Moon TD, at al. Defining the complications of cryoablation and radiofrequency ablation of small renal tumors: a multi-institutional review. J Urol. 2004;172(3):874-877.

42. Uzzo RG, Novick AC, Nephron sparing surgery for renal tumor: indications, techeniques and outcomes. J Urol. 2001;166(1):6-18.

43. Ramani AP, Steinberg AP, Ng CS, Abreu SC, Kaouk JH, Finelli A, et al. Complications of laparoscopic partial nephrectomy in 200 cases. J Urol. 2005; 173(1):42-47.

44. Baker M, Anderson JK, Jaffer O, Trimmer C, Cadeddu JA. Pain after percutaneous radiofrquency ablation of renal tumors. J Endourol. 2007;21(6):606-609.

45. McDougal WS. Radiofrequency ablation of renal cell carcinoma. BJU Int. 2007;99(5 Pt B):1271-1272.

Correspondencia autor: Dr. Javier Estebanez Zarranz Servicio de Urología. Hospital Donostia

Paseo Dr. Beguiristain, s/n - 20014 San Sebastián

Tel.: 943007021

E-mail autor: javier99@hotmail.com

Información artículo: Original

Trabajo recibido: marzo 2009

Trabajo aceptado: abril 2009 